\title{
Nelarabine Regimen
}

National Cancer Institute

\section{Source}

National Cancer Institute. Nelarabine Regimen. NCI Thesaurus. Code C160089.

A chemotherapy regimen consisting of nelarabine that may be used in the treatment of acute lymphoblastic leukemia (ALL). 